\title{
The effect of powder to liquid ratio on physical properties and fluoride release of glass ionomer cements containing pre-reacted spherical glass fillers
}

\author{
Piyaphong PANPISUT ${ }^{1}$, Naruporn MONMATURAPOJ ${ }^{2}$, Autcharaporn SRION ${ }^{3}$, Chayanit ANGKANANUWAT ${ }^{1}$, \\ Nantawan KRAJANGTA ${ }^{1}$ and Prathip PANTHUMVANIT' ${ }^{1}$ \\ ${ }^{1}$ Faculty of Dentistry, Thammasat University, 99 Moo 18, T. Klong1, A. Klongluang, Pathum Thani 12121, Thailand \\ ${ }^{2}$ Assistive Technology and Medical Devices Research Center (A-MED), 111 Thailand Science Park, T. Klong1, A. Klongluang, Pathum Thani 12120, \\ Thailand \\ ${ }^{3}$ National Metal Materials Technology Center (MTEC), 114 Thailand Science Park, T. Klong1, A. Klongluang, Pathum Thani 12120, Thailand \\ Corresponding author, Piyaphong PANPISUT; E-mail: panpisut@staff.tu.ac.th
}

\begin{abstract}
The aim was to assess the effect of powder to liquid ratio (PLR) on setting time, fluoride release, and compressive strength of conventional glass ionomer cements (GICs) containing pre-reacted spherical glass fillers (SPG). GICs were prepared by mixing SPG with Fuji IX Universal liquid using PLR of 1:1, 1.5:1, 2:1, 2.5:1, 3:1. Setting time decreased from 221 to 51 s upon rising PLR. Increasing PLR decreased cumulative fluoride release (33 to $13 \mathrm{ppm}$ ). Diffusion coefficient of fluoride of experimental GICs (1.6$\left.1.8 \times 10^{-8} \mathrm{~cm}^{2} / \mathrm{s}\right)$ was comparable with that of Fuji IX Universal $\left(1.6 \times 10^{-8} \mathrm{~cm}^{2} / \mathrm{s}\right)$. Compressive strength of PLR 2:1 to 3:1 (93-140 MPa) were comparable with that of Fuji IX Universal (124 MPa). These results demonstrated that rising powder ratio reduced setting time, fluoride release, and compressive strength of GICs. However, the setting time and strength experimental GICs with PLR greater than 2:1 were in the acceptable range of the ISO standard.
\end{abstract}

Keywords: Pre-reacted glass, Glass ionomer cement, Compressive strength, Fluoride release, Rheological properties

\section{INTRODUCTION}

World Health Organization (WHO) reported that approximately $60-90 \%$ of adult and almost $100 \%$ of children experienced untreated dental caries ${ }^{1}$. In 2016 , The estimated burden for treatments of caries in the US was almost 124 billion USD ${ }^{2,3}$. The most commonly used restorative materials include dental amalgam, resin composites, and glass ionomer cements (GICs). Dental amalgam is widely used and considered as cost-effective and clinical proven restorative material ${ }^{4)}$. However, the mercury containing products including dental amalgam will be eventually phased down due to the agreement in Minamata Convention on Mercury ${ }^{5}$. Resin composites and dentine adhesives have been substantially improved leading to the excellent mechanical properties in addition to the good esthetics appearance. However, most of the commercial composites were unable to promote remineralizing/antibacterial effects and also required complicating bonding procedures. These shortcomings may lead to bacterial microleakage and secondary caries ${ }^{6}$. It was reported that the high caries risk patients were more susceptible to secondary caries than the low caries risk group ${ }^{7}$.

GICs exhibit attractive properties such as the chemical bond to tooth structure and the ability to release fluoride. These characteristics of the materials may consider suitable for the high caries risk patients or the patients with special needs ${ }^{8,9}$. Conventional GICs however exhibited low mechanical strength ${ }^{10,11}$.

Color figures can be viewed in the online issue, which is available at J-STAGE.

Received Apr 4, 2019: Accepted Jul 16, 2019

doi:10.4012/dmj.2019-097 JOI JST.JSTAGE/dmj/2019-097
The low mechanical properties may contribute to the higher annual failure rate observed with GICs (7\%) compared with that of dental amalgam and resin composites $(\sim 1-3 \%)^{12)}$. One of the common causes of GIC restoration failures was material fracture or chipping ${ }^{13)}$. Monmaturapoj et. al. demonstrated that the addition of pre-reacted spherical glass fillers and irregular particles into conventional GIC significantly improved mechanical properties of the materials ${ }^{14}$. This was probably due to the addition of bimodal particle size that could potentially help increase packing density of the GIC. Additionally, the small particles $(\sim 5-10 \mu \mathrm{m}$ in diameter) had high surface area which may promote the degree of polymer crosslinks. It is believed that these promising fillers may help increase the mechanical properties of GICs.

It is known that varying powder to liquid ratio (PLR) of GICs affected physical properties of the materials. PLR can be varied depending upon the desired clinical applications. Using high PLR usually increases viscosity and strength of GICs which may be suitable in loadbearing areas ${ }^{15}$. Rising PLR also promoted the rapid setting which may beneficial when the ideal moisture isolation or patient cooperation are difficult to obtain. The enhanced mechanical strength of GIC from rising PLR may due mainly to the increase in unreacted particles that act as reinforcing fillers resisting crack propagation within the materials ${ }^{16}$. However, the excessive powder ratio may lead to mixing/handling difficulties. This may increase the risk of air entrapment and generate porosities in the material's bulk, thus reducing strength of the materials ${ }^{17}$. Additionally, high PLR may subsequently lead o the inadequate acid-base 
neutralization which may reduce the release of fluoride. Low viscosity GICs mixed by low PLR may be suitable for protection of tooth surface in caries susceptible areas such as the exposed root surface in patients with hyposalivation ${ }^{18)}$. Using low PLR however led to the prolong setting time and the increase in the risk of moisture contamination that could detrimentally reduce the physical properties of $\mathrm{GICs}^{17)}$. This also reduced radiopacity of the materials ${ }^{19}$. The aim of this study was therefore to assess the effect of different PLR on setting time, fluoride release, and compressive strength of experimental conventional GICs containing pre-reacted spherical glass filler (SPG).

\section{MATERIALS AND METHODS}

Preparation and characterization of spherical pre-reacted glass filler (SPG)

In this study, fluoroaluminosilicate glass system based on the compositions of $\mathrm{SiO}_{2}-\mathrm{Al}_{2} \mathrm{O}_{3}-\mathrm{CaF}_{2}-\mathrm{ZrO}_{2}$ was prepared according to the previously published study ${ }^{14)}$. The glass was produced by mixing $\mathrm{SiO}_{2}$ (Ajax Finches, New South Wales, Australia), $\mathrm{Al}_{2} \mathrm{O}_{3}$ (Fluka, Analytical, Munich, Germany), $\mathrm{P}_{2} \mathrm{O}_{5}$ (Acros Organics, Geel, Belgium), $\mathrm{CaF}_{2}$ (Merck, Darmstadt, Germany), $\mathrm{ZrO}_{2}$ (Sigma-Aldrich, Darmstadt, Germany) and $\mathrm{SrCO}_{3}$ (Sigma-Aldrich) to produce the mixed oxide powders. The powders were melted in a Pt-10\%RH crucible at $1,450^{\circ} \mathrm{C}$ for $2 \mathrm{~h}$. Then the melted glass was rapidly quenched in water to make glass frits. The obtained frits were then reduced the particle size by a planetary micromill (Fritsch Pulverisette, Idar-Oberstein, Germany) by using zirconia balls and deionized water as a grinding medium for $8 \mathrm{~h}$. The X-ray diffraction analysis (XRD; PANalytical, Westborough, MA, USA) was used to determine phase formation of the glass by operating from $20-60^{\circ} 2 \theta$ at a scan speed of $2^{\circ} 2 \theta /$ min and a step size of $0.02^{\circ} 2 \theta$ with $\mathrm{CuK}$ radiation $(\mathrm{K} \alpha=1.5406 \mathrm{~nm})$ at $30 \mathrm{~mA}$ and $50 \mathrm{kV}$. The composition of the glass after melting was additionally analyzed by wavelength dispersive X-ray fluorescence (XRF; ZXS Primus, Rigaku, Japan).

The pre-reacted glass fillers were prepared by mixing the glass powder with 2 wt\% of Fuji IX Universal liquid (GC, Tokyo, Japan). The pilot study revealed that using $2 \mathrm{wt} \%$ of the liquid provided desirable strength for experimental GICs. The mixture was then ground using a ball mill for $3 \mathrm{~h}$ to obtain homogeneous slurry. The slurry was spray-dried using a laboratory scale spray dryer (B-290 Büchi
Mini Spray Dryer, Büchi, Flawil, Switzerland) with an inlet temperature of $200^{\circ} \mathrm{C}$ (outlet temperature of approximately $\left.85-100^{\circ} \mathrm{C}\right)$.

An FTIR (Perkin Elmer System 2000, Perkin Elmer Public, Waltham, MA, USA) was used to detect the acidbase reaction between glass powder and poly(alkenoic acid) liquid in the pre-reacted glass powder. The spectra were obtained in the range of 4,000-400 $\mathrm{cm}^{-1}$ using averaging 20 scans with the resolution of $4 \mathrm{~cm}^{-1}$. The particle size of glass fillers before and after spray drying was also analyzed using the laser diffraction technique (Mastersizer 2000, Malvern instruments, Marlvern, UK) and the scanning electron microscopy (JEOL JSM6301F, JEOL, Tokyo, Japan) respectively. The prereacted glass before (irregular shape) and after spraydrying (spherical shape) were mixed at weight ratio of 60:40 to produce powder phase (SPG) for mixing with liquid phase.

\section{GICs preparations}

Powder phase (SPG) was mixed with the liquid phase of Fuji IX Universal using the PLR of 1:1, 1.5:1, 2:1, 2.5:1, and $3: 1$. The powder and liquid phases were weighted using a four-figure balance and hand-mixed using a plastic spatula for 10-20 s. Commercial conventional GIC (Fuji IX Universal, GC) was used as a comparison. Non-fluoride containing resin composite (Estelite Sigma Quick shade A3, Tokuyama, Tokyo, Japan) was used as a control in fluoride releasing studies. The commercial materials used in the current study are purchased from suppliers (Table 1).

\section{Setting time}

A strain-controlled rheometer (Advanced Rheometric Expansion System, TA Instruments, New Castle, DE, USA) was used to assess the rheological properties of GICs after mixing ${ }^{20)}$. For experimental GICs, the power and liquid were weighed using a four-digit balance. They were then hand-mixed using a plastic spatula for 10-20 s. The commercial GIC was prepared following the manufacturer's instruction. The freshly mixed GICs were then placed between two parallel plate geometries ( $8 \mathrm{~mm}$ in diameter). The gap between two plates was set at $1 \mathrm{~mm}$. The applied strain and oscillatory frequency were maintained at $0.02 \%$ and $1 \mathrm{rad} / \mathrm{s}$ respectively. The temperature was set at $37 \pm 1^{\circ} \mathrm{C}$. Storage modulus (G') of each sample was measured for 5 min (time-sweep measurement). The test was performed in triplicate. Time when G' reached $90 \%$ of their maximum was defined as setting time of the materials ${ }^{20)}$.

Table 1 Composition of commercial materials

\begin{tabular}{lllc}
\hline \multicolumn{1}{c}{ Materials } & Abbreviation & \multicolumn{1}{c}{ Components } & Suppliers \\
\hline $\begin{array}{l}\text { Fuji IX Universal number } \\
\text { (conventional GIC) }\end{array}$ & Fuji IX & $\begin{array}{l}\text { Liquid: polyacrylic acid, Polybasic, polycarboxylic acid } \\
\text { Powder: Fluoro-alumino-silicate glass }\end{array}$ & GC, Tokyo, \\
Estelite Sigma Quick \\
$\begin{array}{l}\text { (resin composite) } \\
\text { Composite }\end{array}$ & & $\begin{array}{l}\text { Zirconia/ silica nanofilled composite, UDMA, } \\
\text { Bis-GMA, TEGDMA }\end{array}$ & $\begin{array}{l}\text { Tokuyama, } \\
\text { Tokyo, Japan }\end{array}$ \\
\hline
\end{tabular}




\section{Fluoride release}

Materials were mixed and placed in a metal circlip (10 $\mathrm{mm}$ in diameter and $1 \mathrm{~mm}$ in thickness) to produce disc specimens $(n=3)$. The specimens were left in an incubator at the controlled temperature of $37^{\circ} \mathrm{C}$ for $24 \mathrm{~h}$. The specimens were then removed and placed in a tube containing $5 \mathrm{~mL}$ of deionized water. At each time points (1-5 days and 1-8 weeks), the storage solution was collected for analysis and replaced with a fresh solution. The collected solution was mixed with TISAB III (Orion ionplus, Thermoscientific, Waltham, MA, USA) using 1:10 volume ratio. Fluoride calibration standards $(0.1,1$, $10,100 \mathrm{ppm}$ ) were prepared using the standard fluoride solution (Orion ionplus, Thermoscientific). Fluoride release was measured using the fluoride specific ion electrode (Orion Versastar Pro, Thermoscientific). The cumulative fluoride ion release was calculated using the following equation.

$$
F_{c}=\Sigma_{0}^{t} F_{t}
$$

Equation 1

Where $F_{c}$ is the cumulative fluoride ion release (ppm), $F_{t}$ is the amount of fluoride (ppm) at time $t$. A modified Fickian diffusion equation (Equation 2) was then employed to assess fluoride releasing kinetics.

$$
\Delta F_{t}=\left(\Delta F_{\max }\right) 2 \sqrt{\frac{D_{t}}{\pi l^{2}}}
$$

Equation 2

Where $\Delta F_{t}$ and $\Delta F_{\max }$ is the cumulative release and the maximum release at 1 week, $D$ is diffusion coefficient $\left(\mathrm{cm}^{2} / \mathrm{s}\right), l$ is specimen thickness $(\mathrm{m})$.

\section{Compressive strength and modulus of elasticity}

Materials were mixed and placed into a cylindrical stainless steel mold $(6 \mathrm{~mm}$ in height and $4 \mathrm{~mm}$ in diameter, $n=6)$. They were left cured in an incubator at the controlled temperature of $37^{\circ} \mathrm{C}$ for $1 \mathrm{~h}$. Then, specimens were removed, trimmed, and immersed in a tube containing $5 \mathrm{~mL}$ of deionized water in an incubator at $37^{\circ} \mathrm{C}$ for $23 \mathrm{~h}$ prior to the compressive strength testing. The test was performed using a universal testing machine (Intron 4502, Wycombe, UK) equipped with a $10 \mathrm{kN}$ load cell at a crosshead speed of $1 \mathrm{~mm} / \mathrm{min}$. Compressive strength $\left(C_{S}, \mathrm{~Pa}\right)$ was calculated using the following equation.

$$
C_{S}=\frac{F}{\pi l^{2}}
$$

Equation 3

Where $F$ is load at failure $(\mathrm{N}), l$ is radius of specimen (m). Additionally, compressive modulus $\left(C_{E}, \mathrm{~Pa}\right)$ was calculated using the following equation.

$$
C_{E}=\frac{F / \pi l^{2}}{\Delta L / L_{0}}
$$

Equation 4

Where $\Delta L$ and $L_{0}$ are change in length (m) and original length $(\mathrm{m})$ of specimens respectively.

\section{Statistical analysis}

All values and errors reported in the current study are mean and 95\% confidence intervals (95\% CI) respectively. SPSS Statistics version 25 for Mac (IBM, Armonk, NY, USA) software was used for statistical analysis. Homogeneity of variance was assessed using Levene's test. When variances were equal, data were analyzed using one-way analysis of variance (ANOVA) followed by the post-hoc Tukey's test for multiple comparisons. Alternatively, the Kruskal-Wallis test followed by multiple comparison using Dunnett's T3 test was used if their variances were not equal. Linear regression analysis between PLR and properties of experimental GICs was also tested. The significance value of all tests was set at $p=0.05$. Furthermore, post hoc power analysis was performed using $G *$ Power version 3.1.9.4 for Mac (University of Dusseldorf, Düsseldorf, Germany). The results indicated that the sample size of each test gave power $>0.99$ at $\alpha=0.05$.

\section{RESULTS}

\section{Characterization of SPG fillers}

XRD pattern demonstrated that the prepared glass was in an amorphous structure with no any minor crystalline formation (Fig. 1A). The glass composition revealed the reduction in the fluoride content of the post-melted glass due to the volatilization of $\mathrm{SiF}_{4}$ during melting and the incrementation of $\mathrm{CaO}$ content (Table 2). The shape of glass fillers without spray drying was irregular with a mean particle diameter of $\sim 5 \mu \mathrm{m}$. After spray drying, the glass formed a loosely agglomerated spherical particle with the mean particle diameter of $\sim 25 \mu \mathrm{m}$. FTIR spectra showed small peaks at 1,600 and 1,450 $\mathrm{cm}^{-1}$ corresponding to antisymmetrical and symmetrical stretching of carboxylate salts respectively ${ }^{21)}$ which indicated the occurrence of acid-base reaction in the SPG fillers (Fig. 1B).

\section{Setting time}

Storage modulus (G') of all materials increased with time (Fig. 2A). The lowest G' after immediate mixing was observed with PLR1:1 whilst the highest G' was observed amongst PLR2:1, PLR2.5:1, and PLR 3:1. After mixing, G' of all materials reached $90 \%$ of their maximum at $5 \mathrm{~min}$ which may represent setting time of the materials. The longest setting time was observed with PLR1:1 (221 \pm 30 s) which was not significantly different from that of Fuji IX $(202 \pm 3 \mathrm{~s}, p=0.888)$ and PLR1.5:1 ( $160 \pm 10 \mathrm{~s}, p=0.217$ ) (Fig. 2B). The shortest setting time was obtained from PLR3:1 (51 1 s). Additionally, the significant correlation between rising PLR and the decrease of setting time was also observed $\left(\mathrm{R}^{2}=0.93, p<0.01\right)$ (Fig. $\left.2 \mathrm{C}\right)$.

\section{Fluoride release}

Fluoride release was detected from all materials except for the dental composite. The release of fluoride from all GICs increased linearly with a square root of hour $\left(\mathrm{hr}^{1 / 2}\right)\left(\mathrm{R}^{2}>0.99\right)$ (Fig. 3A). The fluoride release was not 

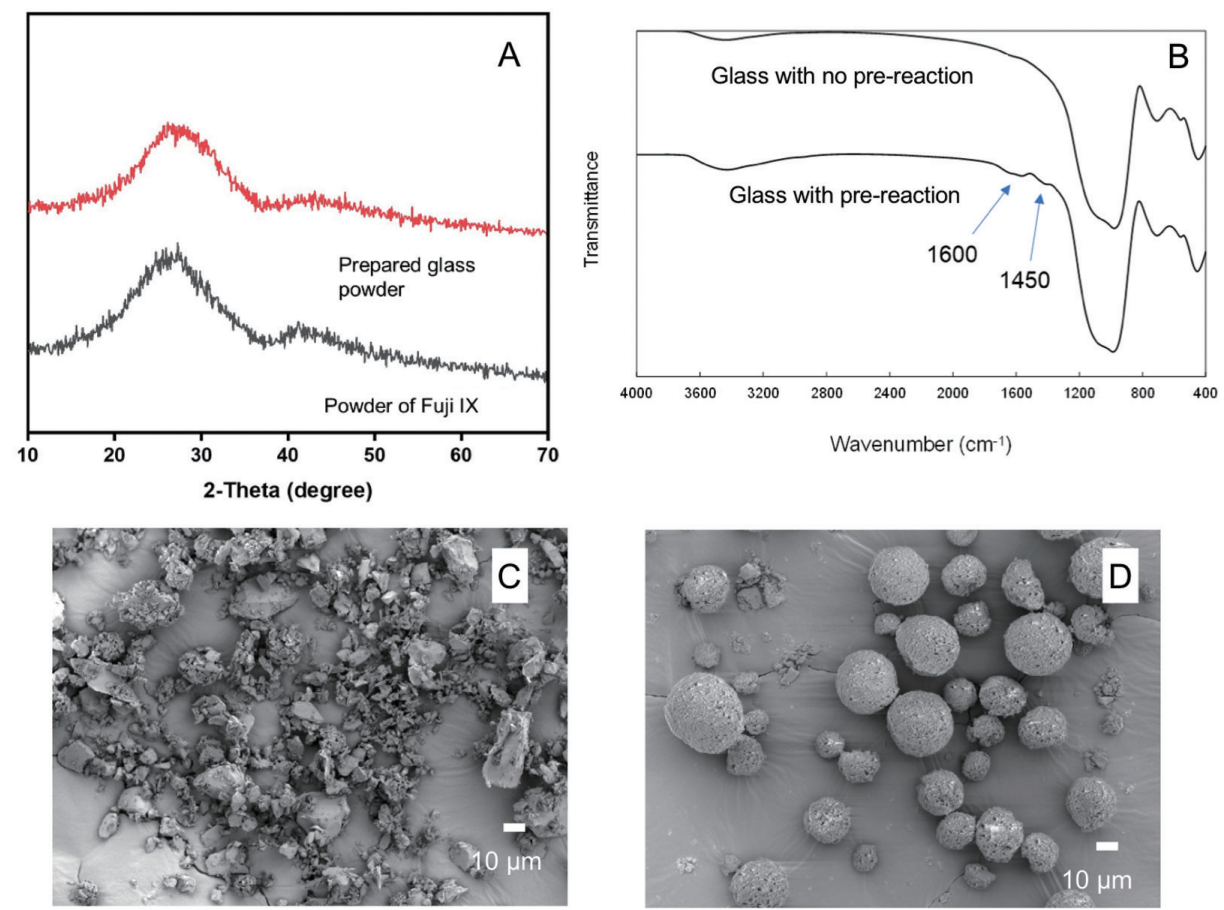

Fig. 1 A) XRD patterns of prepared glass fillers and the powder of Fuji IX Universal.

B) FTIR spectra of glass fillers with and without pre-reaction. SEM images of C) pre-reacted glass fillers before spray drying and D) pre-reacted glass fillers after spray drying.

Table 2 Composition of the prepared glass fillers

\begin{tabular}{lcr}
\hline Oxides & Pre-melted glass compositions (wt\%) & Post-melted glass compositions (wt\%) \\
\hline $\mathrm{SiO}_{2}$ & 22.24 & 20.59 \\
$\mathrm{Al}_{2} \mathrm{O}_{3}$ & 20.59 & 19.01 \\
$\mathrm{P}_{2} \mathrm{O}_{5}$ & 12.77 & 12.92 \\
$\mathrm{CaO}$ & - & 8.37 \\
$\mathrm{SrO}$ & 22.50 & 22.62 \\
$\mathrm{CaF}$ & 14.16 & 10.85 \\
$\mathrm{ZrO}_{2}$ & 6.93 & 5.39 \\
$\mathrm{Total}$ & 99.19 & 99.75 \\
$\mathrm{Al}_{2} \mathrm{O}_{3}: \mathrm{SiO}_{2}$ & 0.93 & 0.92 \\
\hline
\end{tabular}

levelling off at 8 weeks measurement. The highest and lowest diffusion coefficient of fluoride at 1 week was observed with PLR1:1 $\left(1.81 \times 10^{-8} \pm 1.28 \times 10^{-9} \mathrm{~cm}^{2} / \mathrm{s}\right)$

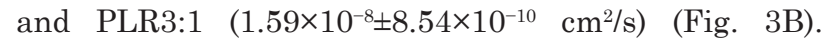
Diffusion coefficient of fluoride was slightly decreased $\left(1 \times 10^{-9} \mathrm{~cm}^{2} / \mathrm{s}, \mathrm{R}^{2}=0.90\right)$ upon raising PLR. The diffusion coefficient of fluoride of all experimental GICS were comparable that of Fuji IX $(p>0.05)$. Additionally, the highest and lowest cumulative fluoride release at

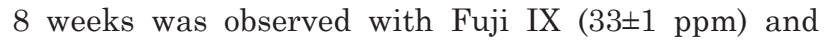
PLR3:1 (13 \pm 1 ppm) respectively (Fig. 3C). Plots of PLR versus diffusion coefficient and cumulative fluoride release in Fig. 3D indicate that the increase of PLR linearly decreased the diffusion coefficient of fluoride $(p=0.03)$ and the cumulative fluoride release $(p<0.01)$.
Compressive strength and compressive modulus

The highest and lowest compressive strength were obtained from PLR2.5:1 (140 $\pm 8 \mathrm{MPa})$ and PLR1:1 (59 \pm 7 MPa) (Fig. 4A). Fuji IX exhibited comparable strength $(124 \pm 23 \mathrm{MPa})$ to PLR2:1 $(93 \pm 19 \mathrm{MPa}) \quad(p=0.079)$, PLR2.5:1 $(p=0.639)$, and PLR3:1 $(116 \pm 13 \mathrm{MPa})(p=0.975)$. The highest and lowest compressive modulus were observed with Fuji IX (5.1 $\pm 0.2 \mathrm{GPa})$ and PLR1:1 (2.2 \pm 0.2 GPa) (Fig. 4B). The modulus of Fuji IX was comparable to that of PLR2.5:1(4.8 $\pm 0.2 \mathrm{GPa})(p=0.910)$ and PLR3:1 $(4.7 \pm 0.5 \mathrm{GPa}) \quad(p=0.435)$. Compressive strength and modulus of the experimental GICs increased linearly upon rising PLR for up to 2.5:1 ( $p<0.01$ ) (Fig. $4 \mathrm{C})$. 


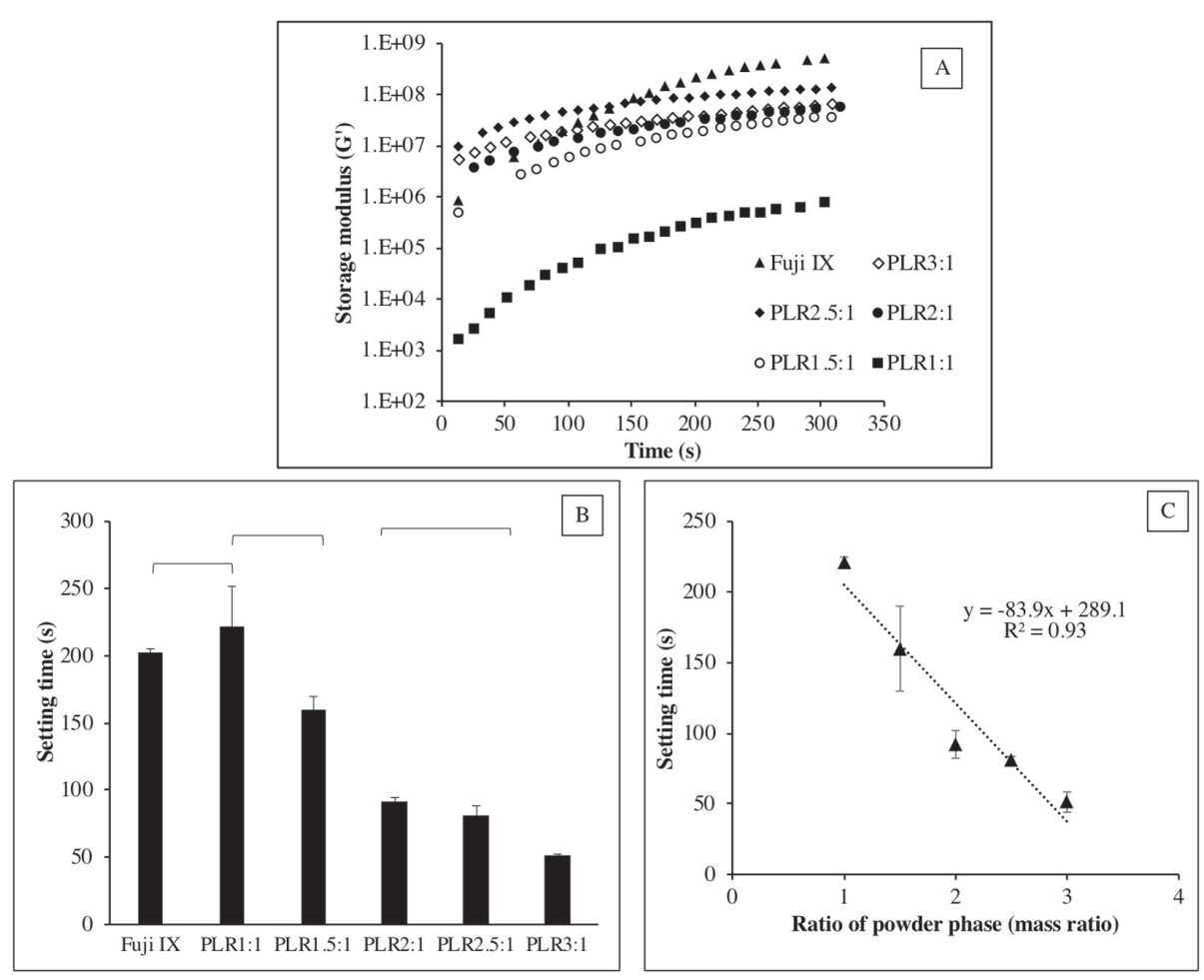

Fig. 2 A) Storage modulus (G') of all GICs after mixing changes with time for up to 5 min. B) setting time of all materials. C) Plot of setting time versus ratio of powder phase. Lines indicate $p>0.05$. Error bars are 95\%CI $(n=3)$.
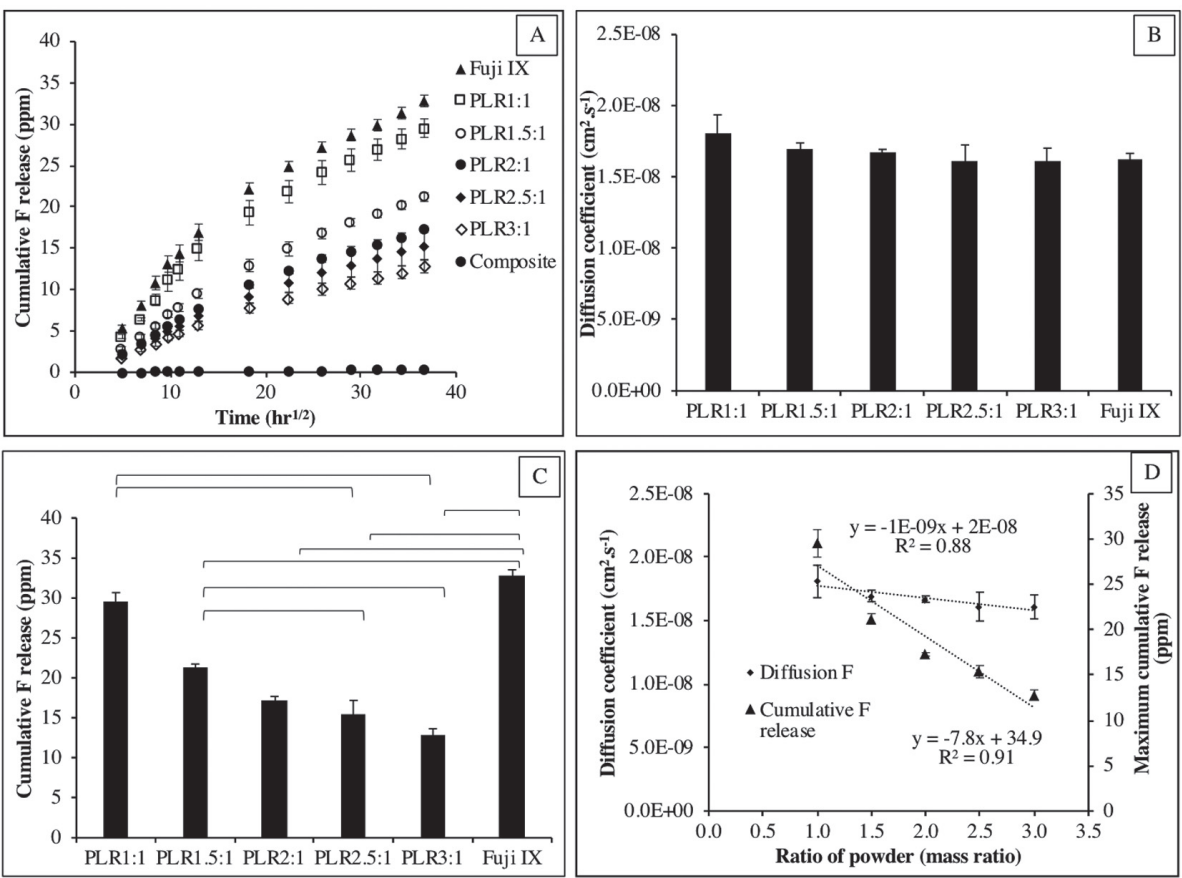

Fig. 3 A) The cumulative fluoride release upon immersion in deionized water for up to 8 weeks plotted against square root of hour. B) Diffusion coefficient of fluoride. C) Total cumulative fluoride release at 8 weeks. D) Plots of diffusion coefficient and maximum cumulative $\mathrm{F}$ release versus powder ratio. Lines indicate significant different $(p<0.05)$. Error bars are 95\% CI $(n=3)$. 


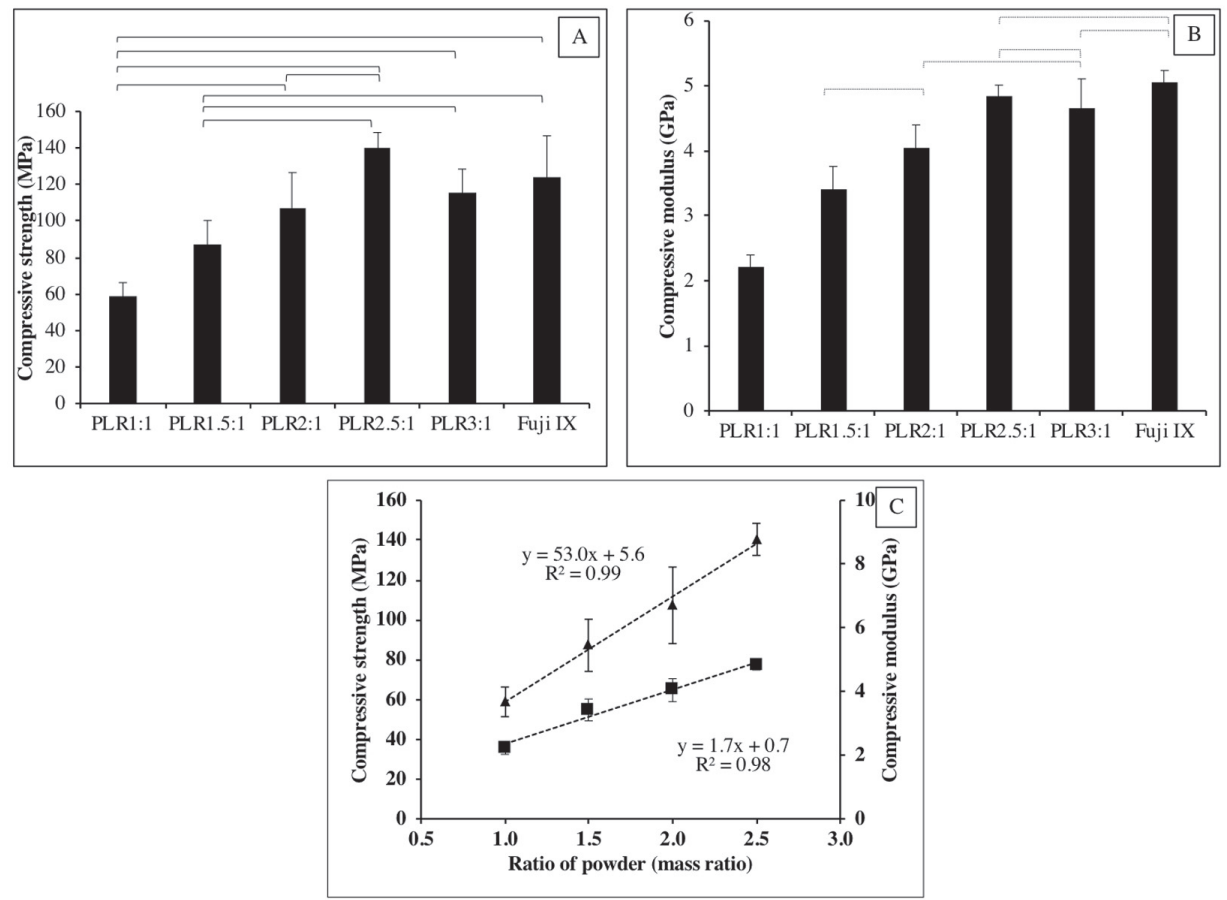

Fig. 4 A) Compressive strength and B) compressive modulus of all materials after immersion in deionized water for $24 \mathrm{~h}$. C) Plots of compressive strength and compressive modulus versus powder ratio (PLR 1:1 to PLR 2.5:1). Error bars are $95 \% \mathrm{CI}(n=6)$. Solid lines and dash lines indicate $p<0.05$ and $p>0.05$ respectively.

\section{DISCUSSION}

This study assessed the effect of PLR of conventional GIC containing previously developed spherical, prereacted glass fillers on setting time, fluoride release, and compressive strength of the materials. The null hypotheses were rejected as rising PLR affected setting time, fluoride release, and strength of the materials.

\section{Setting time}

Storage modulus (G') represents the ability of materials to store energy without a phase difference between stress and strain ${ }^{22}$. G' represents elastic component or the solid-like behavior of fluids. The addition of filler or powder into liquid therefore enhanced solid-like behavior of the materials ${ }^{23}$. Rheological profiles demonstrated the transition from liquid paste to elastic solid (gelation process). A possible explanation of the rising G' would be the formation of crosslinking of polyacrylic acid-chains by $\mathrm{Ca}^{2+}$. This chemical step is believed to be the rate limiting step for the cement hardening ${ }^{24}$.

The setting time obtained from Fuji IX ( 200 s) was within the range reported by the manufacturer (120-360 s) and a published study $(170 \mathrm{~s})^{24)}$. Rising PLR reduced the setting time of GICs as was expected. The rapid setting GICs may be suitable for areas where proper moisture control or the patient compliance are limited. This might be the reason of the development of recent rapid-setting commercial conventional GIC
(Fuji Bulk, GC). The manufacturer reported that the material showed faster initial setting time $(\sim 120$ s) compared with other conventional GICs (120-360 s). It can be seen that the setting time of this new product was comparable with experimental GICs using PLR of 2:1 ( 120 s) and 2.5:1 ( 90 s) given that the hand-mixing time for the materials was approximately $10-20 \mathrm{~s}$. The setting times of GICs using PLR of 2:1 and PLR of 2:5 also in the acceptable range (90-360 s) required by the BS ISO 9917-1 2007: Dentistry-Water-based cements Part 1: Powder/liquid acid-based cement ${ }^{25}$.

\section{Fluoride release}

GICs can release ions such as fluoride, sodium, strontium, aluminium, and calcium ions that may provide buffering effect during acid attack ${ }^{26)}$. The release of fluoride is believed to help promote antibacterial and remineralizing actions ${ }^{27,28)}$. Studies have shown that fluoride release from GICs is governed by several factors such as composition materials, PLR, solubility of glass particles, rate of acid neutralization, water sorption ${ }^{21,299}$. The current study demonstrated the burst release of fluoride at initial time followed by a slow diffusioncontrolled release pattern. This can be explained by the following modified Fickian equation.

$$
\Delta F=\Delta F_{0}+\Delta F_{\infty} \sqrt{\frac{2 D_{t}}{\pi d^{2}}}
$$

Equation 5 
Where $\Delta F$; the change in cumulative fluoride in the solution, $\Delta F_{0}$; early burst release, $\Delta F_{\infty}$; maximum change in the solution, $D$; fluoride diffusion coefficient, $t$; time, $d$; sample thickness. The burst release could be due to rapid surface elution ${ }^{30}$. The subsequent slow fluoride release may be due to the diffusion of fluoride from the slow acid-base neutralization reaction inside the core of materials. All GICs in the current study continued to release fluoride even at the end of measurement. It was reported that the fluoride release from GICs was detected for up to 5 years ${ }^{31,32)}$. The continuation of fluoride diffusion may result from slow acid-base reaction in the bulk of the materials. This may help promote long-term remineralizing actions. Decreasing PLR may accelerating acid-base reaction and increasing the release of fluoride ${ }^{33)}$. This may be of benefit for surface protection of susceptible areas such as the exposed root surface $^{18)}$. However, rising liquid ratio also increased setting time and reduced mechanical properties of the materials.

\section{Compressive strength and modulus of elasticity}

Restorative materials should exhibit high mechanical properties to ensure that the materials can withstand occlusal forces ${ }^{34)}$. In general, the strength of conventional GIC was dominated by polymer matrix with unreacted residual glass fillers that act as reinforcing phase ${ }^{35}$. Low PLR may lead to excessive acid-base reaction that may reduce the amount of residual glass, thus detrimentally reducing strength of the materials ${ }^{16}$.

Compressive strength of Fuji IX obtained from the current study (124 $\pm 23 \mathrm{MPa})$ was comparable with that reported in a published study $(134 \pm 15 \mathrm{MPa})^{36}$. The result from the current study (Fig. 4C) indicated that PLR greater than 1.89 is needed for the experimental GICs to exhibit compressive strength higher than 100 MPa required by the BS ISO 9917-1 200725). However, increasing PLR to 3:1 caused the decline in compressive strength. This could be due to the difficulty in mixing leading the inadequate acid-base neutralization, thus reducing polymer crosslinks ${ }^{15}$. Lowering PLR from 3:1 to 1:1 may accelerate acid-base neutralization reaction enhancing fluoride release. This may however reduce level of unreacted fillers and increase the solubility of materials which could detrimentally affect mechanical strength $^{17)}$. It should be mentioned that the setting specimens were stored in dry condition which may negatively affect their mechanical properties. This could also affect the mechanical properties of the material ${ }^{37}$.

\section{CONCLUSIONS}

The increase of PLR of conventional GICs containing spherical pre-reacted glass filler contain increased compressive strength of the materials. Rising PLR however reduced setting time and the cumulative fluoride release of the experimental GICs. The results demonstrated that using PLR greater than 2:1 enabled acceptable setting time and compressive strength required by the ISO standard.

\section{ACKNOWLEDGMENTS}

The authors would like to express their heartfelt gratitude to Faculty of Dentistry, Thammasat University Research Grant (grant ID: 1/2560) for supporting this study.

\section{CONFLICTS OF INTEREST}

The work is covered by a patent in Thailand of Dr. Naruporn Monmaturapoj (A Method of Making Glass Ionomer Cement, Patent number 1001000006). This does not alter our adherence to Dental Materials Journal policies on sharing data and materials.

\section{REFERENCES}

1) Kassebaum NJ, Bernabe E, Dahiya M, Bhandari B, Murray CJ, Marcenes W. Global burden of untreated caries: a systematic review and metaregression. J Dent Res 2015; 94: 650-658.

2) Correa-Faria P, Daher A, Freire M, de Abreu M, Bonecker M, Costa LR. Impact of untreated dental caries severity on the quality of life of preschool children and their families: a crosssectional study. Qual Life Res 2018; 27: 3191-3198.

3) Martins MT, Sardenberg F, Bendo CB, Abreu MH, Vale MP, Paiva SM, et al. Dental caries remains as the main oral condition with the greatest impact on children's quality of life. PLoS One 2017; 12: e0185365.

4) Austin R, Eliyas S, Burke FJ, Taylor P, Toner J, Briggs P. British society of prosthodontics debate on the implications of the minamata convention on mercury to dental amalgam —should our patients be worried? Dent Update 2016; 43: 8-10, 2-4, 6-8.

5) Mackey TK, Contreras JT, Liang BA. The minamata convention on mercury: attempting to address the global controversy of dental amalgam use and mercury waste disposal. Sci Total Environ 2014; 472: 125-129.

6) Nedeljkovic I, Teughels W, De Munck J, Van Meerbeek B, Van Landuyt KL. Is secondary caries with composites a material-based problem? Dent Mater 2015; 31: e247-277.

7) Opdam NJ, Bronkhorst EM, Loomans BA, Huysmans MC. 12-year survival of composite vs. amalgam restorations. J Dent Res 2010; 89: 1063-1067.

8) Young DA, Frostad-Thomas A, Gold J, Wong A. Secondary Sjogren syndrome: A case report using silver diamine fluoride and glass ionomer cement. J Am Dent Assoc 2018; 149: 731741.

9) Sidhu SK. Glass-ionomer cement restorative materials: a sticky subject? Aust Dent J 2011; 56 Suppl 1: 23-30.

10) Aydinoglu A, Yoruc ABH. Effects of silane-modified fillers on properties of dental composite resin. Mater Sci Eng C Mater Biol Appl 2017; 79: 382-389.

11) Menezes-Silva R, de Oliveira BMB, Fernandes PHM, Shimohara LY, Pereira FV, Borges AFS, et al. Effects of the reinforced cellulose nanocrystals on glass-ionomer cements. Dent Mater 2019; 35: 564-573.

12) Opdam N, Frankenberger R, Magne P. From 'direct versus indirect' toward an integrated restorative concept in the posterior dentition. Oper Dent 2016; 41: S27-S34.

13) Scholtanus JD, Huysmans MC. Clinical failure of class-II restorations of a highly viscous glass-ionomer material over a 6-year period: a retrospective study. J Dent 2007; 35: 156162 .

14) Monmaturapoj N, Soodsawang W, Tanodekaew S. Enhancement effect of pre-reacted glass on strength of glassionomer cement. Dent Mater J 2012; 31: 125-130. 
15) Mulder R. Variation in the dispersions of powder liquid ratios in hand-mix glass ionomers. Open Dent J 2018; 12: 647-654.

16) Torabzadeh H, Ghasemi A, Shakeri S, Baghban AA, Razmavar S. Effect of powder/liquid ratio of glass ionomer cements on flexural and shear bond strengths to dentin. Braz J Oral Sci 2015; 10: 4.

17) Fleming GJ, Farooq AA, Barralet JE. Influence of powder/ liquid mixing ratio on the performance of a restorative glassionomer dental cement. Biomaterials 2003; 24: 4173-4179.

18) Ngo H, Opsahl-Vital S. Minimal intervention dentistry II: part 7. Minimal intervention in cariology: the role of glassionomer cements in the preservation of tooth structures against caries. Br Dent J 2014; 216: 561-565.

19) Fonseca RB, Branco CA, Quagliatto PS, Goncalves Lde S, Soares CJ, Carlo HL, et al. Influence of powder/liquid ratio on the radiodensity and diametral tensile strength of glass ionomer cements. J Appl Oral Sci 2010; 18: 577-584.

20) $\mathrm{Ha} \mathrm{W}$, Nicholson T, Kahler B, Walsh L. Rheological characterization as an alternative method to indentation for determining the setting time of restorative and endodontic cements. Materials 2017; 10: 1451.

21) Young AM. FTIR investigation of polymerisation and polyacid neutralisation kinetics in resin-modified glass-ionomer dental cements. Biomaterials 2002; 23: 3289-3295.

22) Lee IB, Son HH, Um CM. Rheologic properties of flowable, conventional hybrid, and condensable composite resins. Dent Mater 2003; 19: 298-307.

23) Huang HX, Zhang JJ. Effects of filler-filler and polymer-filler interactions on rheological and mechanical properties of HDPE-wood composites. J Appl Polym Sci 2009; 111: 28062812.

24) Algera TJ, Kleverlaan CJ, Prahl-Andersen B, Feilzer AJ. The influence of environmental conditions on the material properties of setting glass-ionomer cements. Dent Mater 2006; 22: 852-856.

25) British Standard. BS EN ISO 9917-1 2007 Dentistry-Waterbased cements. Part 1: Powder/liquid acid-based cement. Brussel: British Standards; 2007.

26) Czarnecka B, Limanowska-Shaw H, Nicholson JW. Buffering and ion-release by a glass-ionomer cement under near-neutral and acidic conditions. Biomaterials 2002; 23: 2783-2788.

27) Tantbirojn D, Feigal RJ, Ko CC, Versluis A. Remineralized dentin lesions induced by glass ionomer demonstrate increased resistance to subsequent acid challenge. Quintessence Int 2006; 37: 273-281.

28) Shahid S, Hassan U, Billington RW, Hill RG, Anderson P. Glass ionomer cements: effect of strontium substitution on esthetics, radiopacity and fluoride release. Dent Mater 2014; 30: 308-313.

29) Yan Z, Sidhu SK, Mahmoud GA, Carrick TE, McCabe JF. Effects of temperature on the fluoride release and recharging ability of glass ionomers. Oper Dent 2007; 32: 138-143.

30) Davis HB, Gwinner F, Mitchell JC, Ferracane JL. Ion release from, and fluoride recharge of a composite with a fluoridecontaining bioactive glass. Dent Mater 2014; 30: 1187-1194.

31) Nicholson JW. Fluoride-releasing dental restorative materials: an update. Balk J Dent Med 2014; 18: 60-69.

32) Asmussen E, Peutzfeldt A. Long-term fluoride release from a glass ionomer cement, a compomer, and from experimental resin composites. Acta Odontol Scand 2002; 60: 93-97.

33) Woranun P, Praphasri R, Rudee S, Kadkao V. Fluoride release from different powder liquid ratios of Fuji VII. Mahidol Dent J 2017; 35: 217-222.

34) Heintze SD, Ilie N, Hickel R, Reis A, Loguercio A, Rousson V. Laboratory mechanical parameters of composite resins and their relation to fractures and wear in clinical trials $-\mathrm{A}$ systematic review Siegward. Dent Mater 2017; 33: E101E14.

35) Alhalawani AMF, Curran DJ, Boyd D, Towler MR. The role of poly(acrylic acid) in conventional glass polyalkenoate cements. J Polym Eng 2016; 36: 221-237.

36) Dowling AH, Fleming GJ, McGinley EL, Addison O. Improving the standard of the standard for glass ionomers: an alternative to the compressive fracture strength test for consideration? J Dent 2012; 40: 189-201.

37) Mitchell CA, Orr JF, Kennedy JG. Factors influencing the failure of dental glass ionomer luting cement due to contraction. Biomaterials 1995; 16: 11-16. 